\title{
Unabsorbed Seyfert 2 galaxies
}

\author{
F. Panessa ${ }^{1,2}$ and L. Bassani ${ }^{1}$ \\ ${ }^{1}$ IASF/CNR, via Piero Gobetti 101, 40129 Bologna, Italy \\ 2 Dipartimento di Astronomia, Universita’ di Bologna, via Ranzani 1, 40127 Bologna, Italy
}

Received 26 March 2002 / Accepted 5 August 2002

\begin{abstract}
We present a sample of 17 type 2 Seyfert galaxies which have an X-ray column density lower than $10^{22} \mathrm{~cm}^{-2}$. The Compton thin nature of these sources is strongly suggested by isotropic indicators. We estimate the fraction of these sources to be in the range of $10 \%-30 \%$ of the population of type 2 Seyfert galaxies. Furthermore, this fraction appears to increase progressively at lower luminosities. The simple formulation of the Unified Model for Seyfert galaxies is not applicable in such sources since the pc-scale molecular torus is not likely to be responsible for the low column density observed; instead the absorption observed is likely to originate at larger scales. According to this hypothesis, in these objects the broad line regions are covered by some dusty obscuring material. In particular, this could occur in objects with dust lanes, patches or HII regions. However, we cannot rule out that in the lowest luminosity sources the BLR is weak, absent or has faded away. This last scenario is consistent with the predictions of some recent theoretical models for low luminosity AGNs.
\end{abstract}

Key words. X-rays: galaxies - galaxies: Seyfert

\section{Introduction}

Seyfert galaxies belong to the class of Active Galactic Nuclei (AGN). According to the standard model, in active galaxies an accretion disk around a massive black hole produces a hard X-ray continuum, which photoionizes the Broad Line Region (BLR, where broad emission lines originate) and the Narrow Line Region (NLR, where narrow emission lines originate) located at $<1 \mathrm{pc}$ and at $<100 \mathrm{pc}$ from the nuclear engine respectively. Seyfert galaxies are classified as type 1 or type 2 . Type 1 have both narrow forbidden lines $\left(F W H M \leq 10^{3} \mathrm{~km} \mathrm{~s}^{-1}\right)$ and broad Balmer lines $\left(F W H M \sim 10^{4} \mathrm{~km} \mathrm{~s}^{-1}\right)$ in their optical spectrum, while type 2 have only narrow lines. Actually they are the same object: type 2 Seyferts harbour a BLR, but this is obscured from view in some directions by a molecular torus (Unification Model; Antonucci 1993).

Optical spectropolarimetry measurements of scattered broad permitted lines provide strong evidence in favour of the unified model (Antonucci \& Miller 1985). At least 35\% of Seyfert 2 galaxies have broad emission lines seen in polarized light (Tran 2001; Moran et al. 2000), therefore a good fraction of Seyfert 2 galaxies seem to host a hidden Seyfert 1 nucleus.

More evidence in favour of the unified model comes from the X-ray spectra: the column density of neutral hydrogen in type 2 Seyferts is significantly higher than in type 1 objects as would be expected if, for the type 2 sources, the nucleus is observed through the torus (Turner et al. 1997; Smith \& Done 1996). Observed column densities range from $10^{22} \mathrm{~cm}^{-2}$ to

Send offprint requests to: F. Panessa, e-mail: panessa@bo.iasf.cnr.it higher than $10^{24} \mathrm{~cm}^{-2}$ for $\sim 96 \%$ of the objects (Risaliti et al. 1999; Bassani et al. 1999).

However, not all Seyfert 2 galaxies have a Broad Line Region in polarized light and not all Seyfert 2 galaxies have column densities higher than $10^{22} \mathrm{~cm}^{-2}$. Polarimetric surveys of complete samples of Seyfert $2 \mathrm{~s}$ indicate that a large fraction of these objects (up to 50\%) do not show a hidden BLR typical of an obscured Seyfert 1 nucleus. Furthermore there have been some recent examples of Seyfert 2 galaxies, such as NGC 3147 (Ptak et al. 1996), NGC 4698 (Pappa et al. 2001) and NGC 7590 (Bassani et al. 1999), which have no or low absorption measured from the X-ray spectrum. It can be argued that these are Compton thick objects i.e. in which the medium is thick to Compton scattering such that the transmitted component is completely suppressed below $10 \mathrm{keV}$ and the $2-10 \mathrm{keV}$ spectrum is dominated by reprocessed components. In this case the hard X-ray spectrum is characterized by a flat Compton reflection component from the inner surface of the torus and/or a steeper component ascribed to an ionized, warm scattering medium. When the absorbing medium has column density $N_{\mathrm{H}}>10^{24} \mathrm{~cm}^{-2}$, then the transmitted component can be observed above $10 \mathrm{keV}$. Therefore, in these sources the true column density can only be estimated by higher energy data for $N_{\mathrm{H}}>10^{24} \mathrm{~cm}^{-2}$ or measured indirectly by comparing the X-ray luminosity with the Far-Infrared or [OIII] luminosities for even higher column densities. In the above mentioned sources these absorption indicators suggest that they are actually Compton thin objects. At the moment at least $\sim 4 \%$ of Seyfert $2 \mathrm{~s}$ have $N_{\mathrm{H}}<10^{22} \mathrm{~cm}^{-2}$ (Risaliti et al. 1999). The exact nature of these peculiar Seyfert $2 \mathrm{~s}$ is still unclear, as it is not 
Table 1. Unobscured Seyfert 2 galaxies sample.

\begin{tabular}{|c|c|c|c|c|}
\hline Nome & RA \& Dec (2000) & $z$ & $N_{\mathrm{HGal}}^{\S}$ & $\begin{array}{l}\text { NED } \\
\text { class. }\end{array}$ \\
\hline IRAS 00317-2142 & $003413.8-212621$ & 0.02680 & 1.5 & $\mathrm{~S} 1.8$ \\
\hline IRAS 01428-0404 & $004525.2-034936$ & 0.01820 & 4.28 & S2 \\
\hline IC 1631 & $010844.8-462833$ & 0.03084 & 2.17 & $\mathrm{~S} 2^{\star}$ \\
\hline NGC 2992 & $094542.0-141935$ & 0.00771 & 5.26 & S2 \\
\hline NGC 3147 & $101653.6+732403$ & 0.00941 & 3.64 & S2 \\
\hline NGC 4565 & $123620.8+255916$ & 0.00428 & 1.30 & S1.9 \\
\hline NGC 4579 & $123743.5+114905$ & 0.00507 & 2.47 & $\mathrm{~L} / \mathrm{S} 1.9$ \\
\hline NGC 4594 & $123958.8-113728$ & 0.00364 & 3.77 & $\mathrm{~L} / \mathrm{S} 1.9$ \\
\hline NGC 4698 & $124823.0+082914$ & 0.00334 & 1.87 & S2 \\
\hline NGC 5033 & $131327.3+363536$ & 0.00292 & 1.03 & S1.9 \\
\hline MRK 273x & $134447.4+555411$ & 0.45800 & 1.10 & S2 \\
\hline NGC 5995 & $154824.9-134528$ & 0.02519 & 10.6 & S2 \\
\hline NGC 6221 & $165246.1-591307$ & 0.00494 & 15.0 & $\mathrm{~S} 2^{\star}$ \\
\hline NGC 6251 & $163231.8+823216$ & 0.02302 & 5.49 & $\mathrm{~S} 2$ \\
\hline IRAS 20051-1117 & $200751.3-110833$ & 0.03149 & 6.8 & $\mathrm{SB} / \mathrm{S} 2$ \\
\hline NGC 7590 & $231855.0-421417$ & 0.00532 & 1.96 & S2 \\
\hline NGC 7679 & $232846.8+033041$ & 0.01714 & 5.13 & $\mathrm{~S} 2^{\star}$ \\
\hline
\end{tabular}

$\S$ Column density in units of $10^{20} \mathrm{~cm}^{-2}$.

$\star$ Transition objects between Seyfert 2 and starburst (see Fig. 1).

obvious what obscures their Broad Line Region: they may be intrinsically different objects than those explained by the unified theory or in other words they may be the "true" Seyfert 2 galaxies which are sometimes discussed in the literature (Tran 2001).

In this paper we have collected a sample of Seyfert 2 galaxies characterized by low X-ray absorption in order to study their properties, estimate their abundance and understand better their nature.

\section{The sample}

The sample consists of objects which are classified in NED as type 2 Seyferts ${ }^{1}$ and are characterized by low absorption in $\mathrm{X}$-rays $\left(N_{\mathrm{H}} \lesssim 10^{22} \mathrm{~cm}^{-2}\right)$.

The 17 objects of the sample are listed in Table 1 with optical positions in equatorial coordinates for epoch $\mathbf{J} 2000$, redshift $z$ as reported in NED, Galactic column density from $21 \mathrm{~cm}$ measurement in units of $10^{20} \mathrm{~cm}^{-2}$ obtained from the HEASARC (High Energy Astrophysics Science Archive Research Center) on-line service and NED classification ( $\mathrm{S}=$ Seyfert, L = Liner, SB = Starburst). In Table 2 we list the main X-ray spectral parameters in addition to the Infrared and [OIII] $\lambda 5007$ flux values.

\footnotetext{
${ }^{1}$ In type 2 optical classification we also include Seyferts 1.9 and 1.8.
}
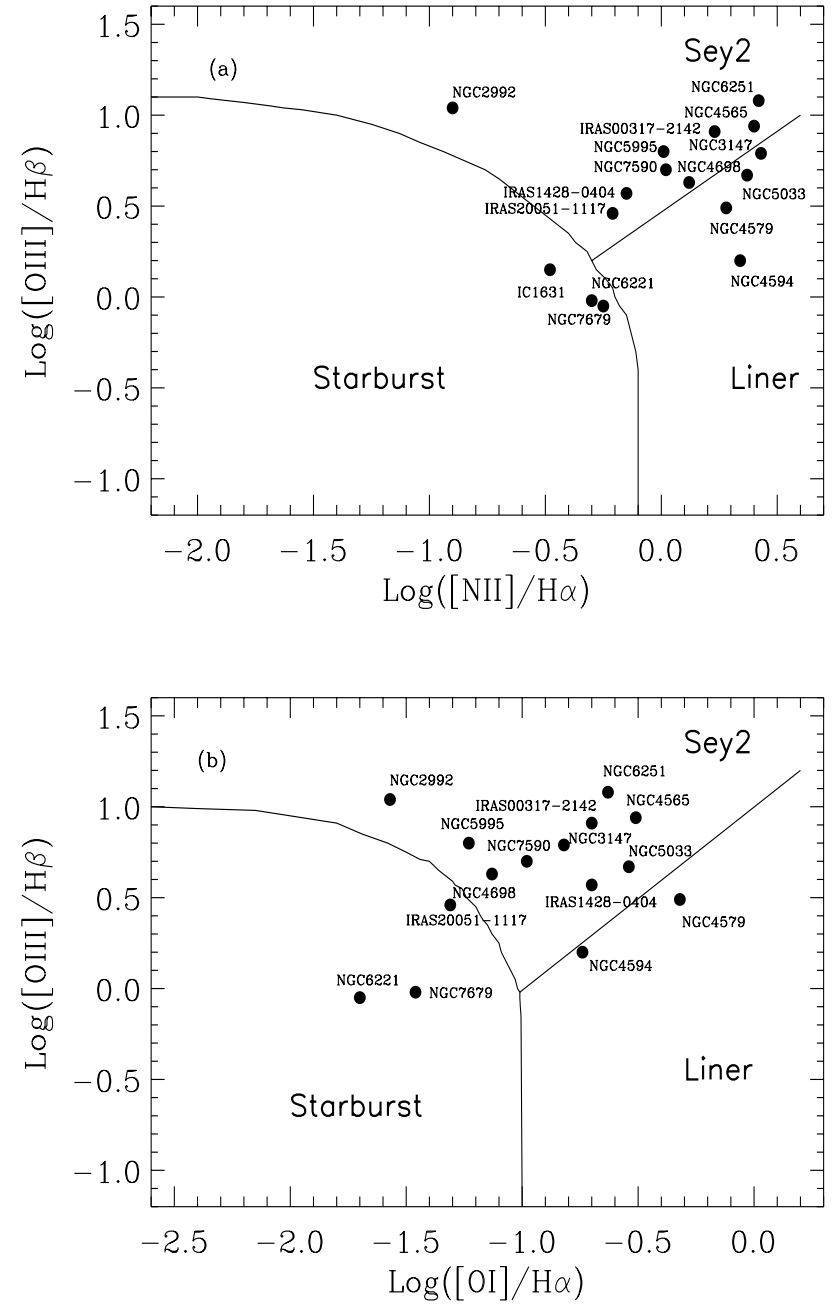

Fig. 1. a) Diagnostic diagram with $[\mathrm{OIII}] \lambda 5007 / \mathrm{H}_{\beta}$ vs. $[\mathrm{NII}] \lambda 6583 / \mathrm{H}_{\alpha}$. b) Diagnostic diagram with $[\mathrm{OIII}] \lambda 5007 / \mathrm{H}_{\beta}$ vs. $[\mathrm{OI}] \lambda 6300 / \mathrm{H}_{\alpha}$. In both a) and b) diagrams Seyfert 2, Starburst and HII regions are divided by solid lines.

\subsection{Optical classification}

All objects in Table 1 are reported as pure or composite Seyfert $2 s$ in NED. In order to check the NED Seyfert 2 classification accurately, we have employed the two optical diagnostic diagrams by Veilleux \& Osterbrock (1987) which use line-intensity ratios that are relatively insensitive to reddening and are considered good excitation indicators. Line ratios such as $[\mathrm{OIII}] \lambda 5007 / \mathrm{H}_{\beta}$, [NII $] \lambda 6583 / \mathrm{H}_{\alpha}$, [OI $] \lambda 6300 / \mathrm{H}_{\alpha}$ delineate the different excitation mechanisms which operate in HII regions, high-excitation AGNs (Seyferts) and low-excitation AGNs (low-ionization nuclear emission-line regions, LINERS; Heckman 1980).

Although the boundaries between these three classes are not rigorously defined, these diagrams represent a valid system to distinguish between various types of narrow emission line objects. The line ratios shown in Fig. 1 are taken from the literature (see the [OIII] $\lambda 5007$ flux references of Table 2). For those sources with more than one observation we adopted the most recent reference; note that not in all cases are the data available or complete. 
Table 2. X-ray spectral parameters for the sample.

\begin{tabular}{|c|c|c|c|c|c|c|c|c|}
\hline & Nome & $\Gamma$ & $N_{\text {Hint }}^{a}$ & $E W\left(\mathrm{Fe}_{\mathrm{k} \alpha}^{b}\right)$ & $F_{2-10 \mathrm{keV}}^{c}$ & $L_{2-10 \mathrm{keV}}^{d}$ & $F_{\mathrm{IR}}^{e}$ & $F_{[\mathrm{OIII}]}^{f}$ \\
\hline 1 & IRAS 00317-2142 & $2.00_{-0.07}^{+0.07}$ & $1.9_{-0.2}^{+0.2}$ & $<900$ & 0.08 & 42.01 & 25.94 & 24 \\
\hline 2 & IRAS 01428-0404 & $1.95_{-0.73}^{+1.33}$ & $32_{-32}^{+109}$ & - & 0.04 & 41.38 & $<7.42$ & - \\
\hline 3 & IC 1631 & $2.10_{-0.10}^{+0.10}$ & $<31.6$ & $<70$ & 1.00 & 43.23 & $<6.85$ & 52.0 \\
\hline 4 & NGC 2992 & $1.70_{-0.2}^{+0.2}$ & $90.0_{-3.0}^{+3.0}$ & $147_{-37}^{+37}$ & 7.4 & 42.30 & 50.79 & 680.0 \\
\hline 5 & NGC 3147 & $1.94_{-0.19}^{+0.20}$ & $<2.9$ & $675_{-328}^{+395}$ & 0.22 & 41.62 & 44.39 & 9.0 \\
\hline 6 & NGC 4565 & $1.7_{-0.2}^{+0.2}$ & $N_{\mathrm{H}, \mathrm{gal}}$ & - & 0.02 & 39.32 & 42.03 & 6.0 \\
\hline 7 & NGC 4579 & $1.88_{-0.03}^{+0.03}$ & $N_{\mathrm{H}, \text { gal }}$ & - & 0.52 & 41.23 & 38.37 & 9.0 \\
\hline 8 & NGC 4594 & $1.5_{-0.03}^{+0.04}$ & $17_{-9}^{+11}$ & - & 0.16 & 40.86 & 31.90 & 7.0 \\
\hline 9 & NGC 4698 & $1.91_{-0.14}^{+0.14}$ & $8.1_{-7.8}^{+8.2}$ & $<425$ & 0.10 & 40.50 & $<3.14$ & 2.4 \\
\hline 10 & NGC 5033 & $1.7_{-0.02}^{+0.02}$ & $N_{\mathrm{H}, \mathrm{gal}}$ & $100_{-100}^{+100}$ & 0.28 & 41.04 & 82.77 & 17.0 \\
\hline 11 & MRK 273x & $1.66_{-0.11}^{+0.15}$ & $14.1_{-5.0}^{+5.5}$ & $<30$ & 0.01 & 43.62 & - & 0.14 \\
\hline 12 & NGC 5995 & $1.81_{-0.03}^{+0.04}$ & $90_{-3}^{+5}$ & $144_{-41}^{+41}$ & 2.89 & 43.52 & 28.54 & 66 \\
\hline 13 & NGC 6221 & 1.9 & $110_{-8.3}^{+8.6}$ & $360_{-93}^{+210 g}$ & 1.4 & 41.78 & - & 2.14 \\
\hline 14 & NGC 6251 & $1.83_{-0.18}^{+0.21}$ & $75_{-58}^{+64}$ & $443_{-272}^{+313 g}$ & 0.14 & 41.13 & - & 57.0 \\
\hline 15 & IRAS 20051-1117 & $1.92_{-0.14}^{+0.21}$ & $<40$ & $272_{-73}^{+52}$ & 0.24 & 42.63 & 7.59 & 15.2 \\
\hline 16 & NGC 7590 & $2.29_{-0.13}^{+0.20}$ & $<9.2$ & - & 0.12 & 40.79 & 44.53 & 17.0 \\
\hline 17 & NGC 7679 & $1.75_{-0.06}^{+0.03}$ & $2.2_{-1.4}^{+1.5}$ & $<200$ & 0.60 & 42.53 & 49.60 & 108.26 \\
\hline
\end{tabular}

${ }^{a}$ Column density in units of $10^{20} \mathrm{~cm}^{-2},{ }^{b}$ Equivalent width of the $\mathrm{Fe}_{k \alpha}$ in units of $\mathrm{eV},{ }^{c} 2-10 \mathrm{keV}$ flux in units of $10^{-11} \mathrm{erg} \mathrm{cm}^{-2} \mathrm{~s}^{-1},{ }^{d} 2-10 \mathrm{keV}$ logarithm of unabsorbed luminosity $\left(H_{0}=75 \mathrm{~km} \mathrm{~s}^{-1} \mathrm{Mpc}^{-1}\right),{ }^{e}$ Infrared flux in units of $10^{-11} \mathrm{erg} \mathrm{cm}^{-2} \mathrm{~s}^{-1},{ }^{f}$ [OIII] $\lambda 5007 \mathrm{corrected} \mathrm{flux} \mathrm{in}$ units of $10^{-14} \mathrm{erg} \mathrm{cm}^{-2} \mathrm{~s}^{-1},{ }^{g}$ Line at $6.65 \mathrm{keV}$. X-ray references: (1) Georgantopoulos et al. (2000); (2) This work, ASCA data; (3) Awaki et al. (1992); (4) Gilli et al. (2000); (5) This work, SAX data; (6) Cappi et al. (2002); (7) Eracleous et al. (2001); (8) Pellegrini et al. (2002); (9) Pappa et al. (2001); (10) Cappi et al. (2002); (11) Xia et al. (2002); (12) This work, ASCA data; (13) Levenson et al. (2001b); (14) Sambruna et al. (1999); (15) This work, ASCA data; (16) Bassani et al. (1999); (17) Della Ceca et al. (2001). All $F_{\text {IR }}$ are based on IRAS data taken from NED. [OIII] references: (1) Moran et al. (1996); (2) Pietsch et al. (1998); (3) Sekiguchi et al. (1993); (4) Gilli et al. (2000); (5) Ho et al. (1997); (6) Ho et al. (1997); (7) Ho et al. (1997); (8) Ho et al. (1997); (9) Ho et al. (1997); (10) Ho et al. (1997); (11) Xia et al. (1999); (12) Lumsden et al. (2001); (13) Levenson et al. (2001b); (14) Shuder \& Osterbrock (1981); (15) Moran et al. (1996); (16) Vaceli et al. (1997); (17) Kewley et al. (2000).

Most of the objects plotted in Fig. 1 show a well defined optical classification: they are classical type 2 sources. As expected, a few objects are located at the boundaries between Seyfert 2 and Liner/Starburst and so are likely to be Composite objects. The composite Seyfert/Liner nature of NGC 4579 and NGC 4594 is clear cut as seen in both diagrams confirming their NED classification; NGC 5033 and NGC 3147 are much less clear examples as they lie at the boundaries in one diagram but not in the other and so we maintain their NED definition.

Transition objects between Seyfert 2 and Starburst are NGC 6221 and NGC 7679; IC 1631 could be similar but unfortunately we lack information on the $[\mathrm{OI}] / \mathrm{H}_{\alpha}$ ratio to confirm this hypothesis. IRAS 20051-1117 which is classified as composite in NED is confirmed as this type only in one of the two diagrams and even in this case it is a borderline object: we therefore take this as an indication of the predominance of the Seyfert 2 signature. Therefore we substantially confirm the NED classifications (except in three cases which are flagged in Table 1) and conclude that all sources of our sample are characterized by an optical type-2 signature.

\subsection{Diagnostic diagrams}

The X-ray characteristics of the sample sources (described in Table 2) strongly suggest the presence of an AGN often of 


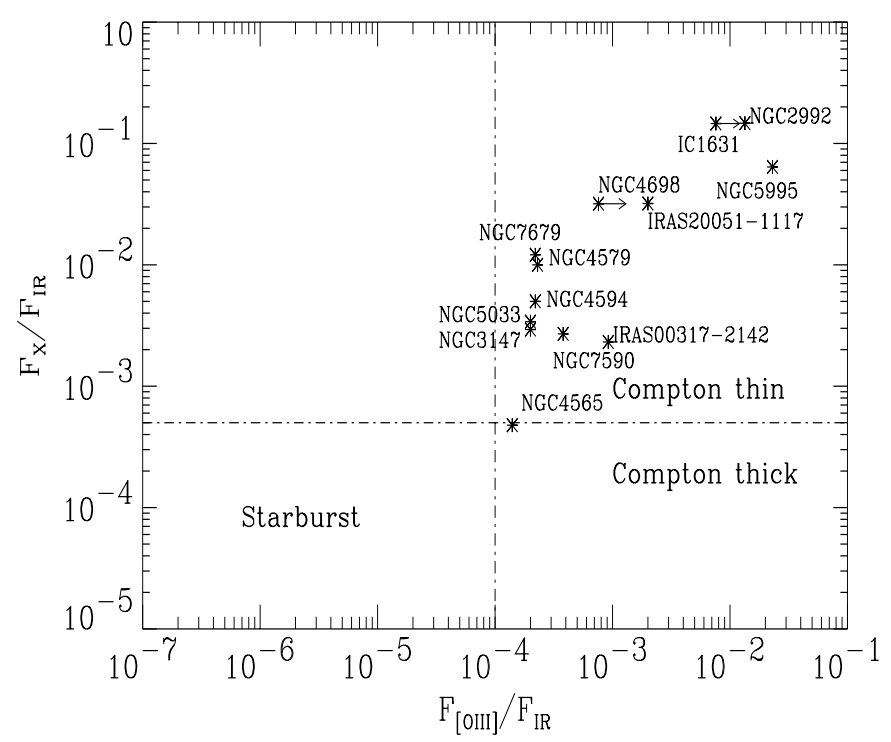

Fig. 2. $F_{\mathrm{X}} / F_{\mathrm{IR}}$ vs. $F_{[\mathrm{OIII}]} / F_{\mathrm{IR}}$ for the sources of the sample. We separate with dashed lines the Compton thin, Compton thick and Starburst region. The fluxes used are listed in Table 2.

low-luminosity in most objects (the photon indices are canonical and Iron lines are sometimes detected). However this evidence is not sufficient to establish the presence of an active nucleus in all objects and in particular in low luminosity sources, where the luminosity does not allow us to discriminate between emission from an active nucleus or a starburst galaxy. Furthermore our objects could be Compton thick $\left(N_{\mathrm{H}}>\right.$ $10^{24} \mathrm{~cm}^{-2}$ ) but since the photoelectric cut-off would not be detectable in the $2-10 \mathrm{keV}$ spectrum, the column density measurements would be too low. However the presence of an AGN and the Compton nature (thin or thick) of each source can be checked by comparing isotropic versus anisotropic properties.

If a molecular torus is present in Seyfert galaxies, then it should block the X-ray emission coming from the central engine but it shouldn't intercept emission coming from larger scale structures like the Narrow Line Region or a non nuclear starburst region. The column density could then be inferred from the flux ratios of the $\mathrm{X}$-ray fluxes versus various isotropic emission measurements. The [OIII] $\lambda 5007$ flux is considered a good isotropic indicator because it is produced in the Narrow Line Region (Maiolino \& Rieke 1995; Risaliti et al. 1999; Bassani et al. 1999). Also the Far-Infrared emission seems to be produced over a larger region than that of the molecular torus and it has been used as an isotropic indicator too.

The $F_{\mathrm{X}} / F_{\text {[OIII] }}$ ratio has been studied in a large sample of Seyfert 2 galaxies: all Compton thin Seyferts show ratios higher than $\sim 1$ while Compton thick sources show ratios below this value (Bassani et al. 1999). The $F_{\mathrm{X}} / F_{\mathrm{IR}}$ ratio has also been largely discussed and used in the literature to investigate the presence of high column densities. Typically type 1 and Compton thin type $2 \mathrm{AGN}$ show ratios of $\sim 0.1$, while Compton thick type 2 objects show ratios lower than $5 \times 10^{-4}$ (David et al. 1992; Mulchaey et al. 1994; Risaliti et al. 1999). Finally, since infrared emission is associated mainly with star-forming activity while the $[\mathrm{OIII}] \lambda 5007$ emission is produced by photons

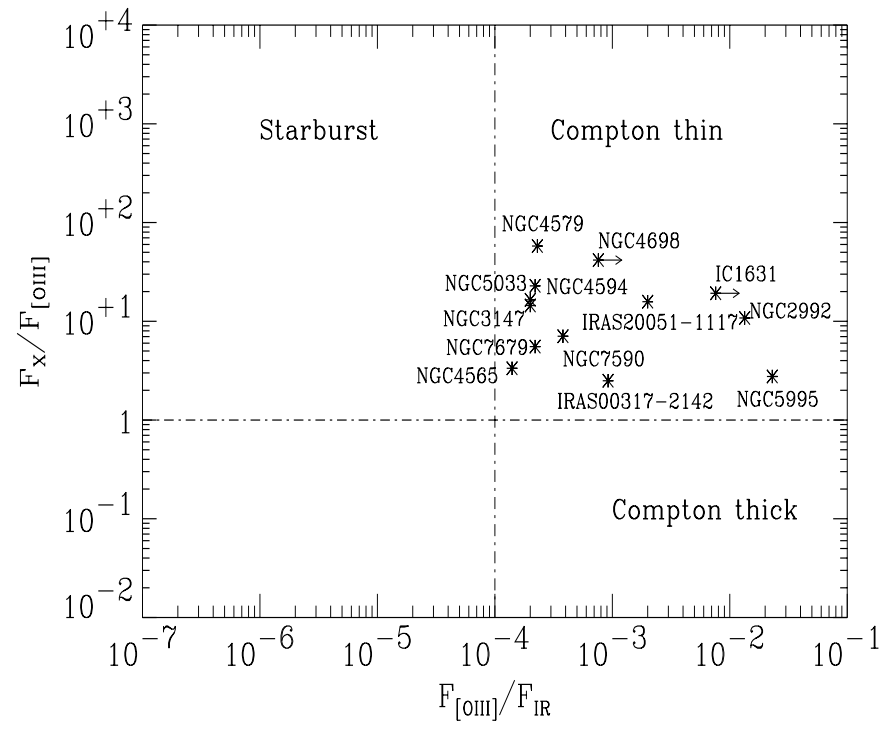

Fig. 3. $F_{\mathrm{X}} / F_{\text {[OIII] }}$ vs. $F_{[\mathrm{OIII}]} / F_{\mathrm{IR}}$ for the sources of the sample. We separate with dashed lines the Compton thin, Compton thick and starburst region. The fluxes used are listed in Table 2.

generated in the active nucleus, by comparing the $F_{[\mathrm{OIII}]} / F_{\mathrm{IR}}$ ratio of all the starbursts and AGNs in the Ho et al. (1997) sample, Bassani et al. (in preparation) find that $\sim 90 \%$ of the starburst galaxies show a value below $10^{-4}$ while $\sim 88 \%$ of AGNs show a value above $10^{-4}$, therefore they suggest this value as a means of discriminating between one galaxy class and the other. In conclusion, these 3 ratios can provide an independent way to establish which is the dominant component between AGN or starburst and at the same time they are a powerful tool in the detection of Compton thick sources when an $\mathrm{X}$-ray spectral analysis is not sufficient.

The fluxes used in our diagrams (Figs. 2 and 3) are listed in Table 2. All far-infrared fluxes are based on IRAS data, taken from NED. For this paper we adopt the same definition used in Mulchaey et al. (1994) for the far-infrared flux:

$F_{\mathrm{IR}}=F_{25 \mu \mathrm{m}} \times v_{25 \mu \mathrm{m}}+F_{60 \mu \mathrm{m}} \times v_{60 \mu \mathrm{m}}$.

Note that due to the IRAS angular resolution the $F_{\text {IR }}$ value is likely to be overestimated for the nuclear region of each galaxy; this translates into $F_{\mathrm{X}} / F_{\mathrm{IR}}$ and $F_{\text {[OIII] }} / F_{\mathrm{IR}}$ ratios smaller than in reality. The $[\mathrm{OIII}] \lambda 5007$ flux of each galaxy has been corrected for extinction using the formula given in Bassani et al. (1999).

In both Figs. 2 and 3 the starburst region is empty suggesting that the dominant component is likely to be an AGN; the region populated by our sources is that of the Compton thin regime, indicating that indeed we measure the real amount of absorption in all our objects. This result is confirmed also for those objects for which the data are not complete: the $F_{\mathrm{X}} / F_{\text {[OIII] }}$ ratios for MRK 273x, NGC 6221 and NGC 6251 are 71,654 and 24 respectively and the $F_{\mathrm{X}} / F_{\mathrm{IR}}$ ratio for IRAS 01428-0404 is $5.4 \times 10^{-3}$.

The major problem when dealing with low luminosity AGN is the possible contamination from off nuclear sources in the observed galaxy: in this case the measured flux is overestimated by non-imaging/low-angular resolution instruments and 
the spectrum alterated. This is what is emerging from recent works based on Chandra and XMM-Newton observations: the $\mathrm{X}$-ray fluxes are in most cases lower than those measured by past satellites (see Chandra results by Ho et al. 2001). A consequence of this could be a mistaken evaluation of the column density and/or of the Compton nature of the source. However, for those 5 sources of our sample which have Chandra or XMM-Newton data the spectral parameters are substantially in agreement with the old values and even if the $2-10 \mathrm{keV}$ luminosities are somehow decreased, all these objects are still in the Compton thin region.

In any case, it is important to consider the possibility that more accurate and higher spatial resolution observations could reduce the flux of some of our sources.

\section{How many unabsorbed Seyfert 2 galaxies are there?}

Bassani et al. (1999) presented a column density distribution for a sample of Seyfert 2 s for which X-ray data were available. The distribution was shifted toward high $N_{\mathrm{H}}$ values and only $\sim 20 \%$ of the sources had a column density less than $10^{22} \mathrm{~cm}^{-2}$. However, the sample used by Bassani et al. (1999) was a collection of data and so it did not fulfill the requirements of completeness. When a complete sample was considered (Risaliti et al. 1999) the column density distribution found was similar for high column density values: $75 \%$ of type 2 Seyferts were found to be heavily obscured $\left(N_{\mathrm{H}}>10^{23} \mathrm{~cm}^{-2}\right)$ with $50 \%$ of the sources being Compton thick $\left(N_{\mathrm{H}}>10^{24} \mathrm{~cm}^{-2}\right)$. However, only $\sim 4 \%$ of the sample was characterized by low absorption $\left(N_{\mathrm{H}}<10^{22} \mathrm{~cm}^{-2}\right)$. The sample considered by Risaliti et al. (1999) was derived by the Maiolino \& Rieke (1995) sample completed with NGC 1808 and integrated with 18 new sources found by Ho et al. (1997) which would have been included in the Maiolino \& Rieke (1995) sample if they had been discovered earlier. This sample has been limited in [OIII] flux $\left(F_{\text {[OIII] }}>40 \times 10^{-14} \mathrm{erg} \mathrm{cm}^{-2} \mathrm{~s}^{-1}\right)$ in order to be independent of absorption effects and to have the greatest possible range in column density.

We have reconsidered the total sample reported in Risaliti et al. (1999), considering also the sources with $F_{\text {[OIII] }}<40 \times$ $10^{-14} \mathrm{erg}^{-2} \mathrm{~s}^{-1}$ and we have updated the column density distribution with $N_{\mathrm{H}}$ measurements which have become available in the meantime. Finally, we have used the X-ray versus [OIII] flux ratios to assess the possible Compton thick nature of the sample sources, if a column density was not available: sources found to be Compton thick were ascribed to $N_{\mathrm{H}}>10^{24} \mathrm{~cm}^{-2}$ range. This sample consists of 92 sources and for 59 sources we have the value of the column density. We find that the fraction of objects with $N_{\mathrm{H}}<10^{22} \mathrm{~cm}^{-2}$ increases to $\sim 12 \%$ and this is to be considered a lower limit as not all sources have the column density measured. If we consider only the 59 sources with known $N_{\mathrm{H}}$, the fraction is $\sim 18 \%$.

In order to check this result we also considered all sources which are classified as type 2 Seyferts (including the transition objects indicated as Liner2/Seyfert2 or Transition2/Seyfert2) in the Ho et al. (1997) sample, which is complete in magnitude to $B_{\mathrm{T}}<12.0 \mathrm{mag}$. The total number of these objects is 49
Table 3. Fractions for three samples.

\begin{tabular}{lccc}
\hline \hline $\begin{array}{l}\log \left(N_{\mathrm{H}}\right) \\
\mathrm{cm}^{-2}\end{array}$ & $\begin{array}{l}\text { Risaliti (99) } \\
\text { total sample }\end{array}$ & $\begin{array}{c}\text { Ho et al. (97) } \\
\text { sample }\end{array}$ & $\begin{array}{c}\text { Ho sample } \\
<22 \mathrm{Mpc}\end{array}$ \\
\hline$<22$ & $>12 \%$ & $>10 \%$ & $>15 \%$ \\
$22-23$ & $>14 \%$ & $>4 \%$ & $>7 \%$ \\
$23-24$ & $>16 \%$ & $>14 \%$ & $>19 \%$ \\
$>24$ & $>22 \%$ & $>10 \%$ & $>11 \%$ \\
no data & $36 \%$ & $62 \%$ & $48 \%$ \\
\hline
\end{tabular}

References for $N_{\mathrm{H}}$ : Ho (1999), Allen et al. (2000), Terashima et al. (2000), Guainazzi (2002), Pappa et al. (2001), Levenson et al. (2001b), Cappi et al. (2002).

and the column density has been determined for 19 of them. For all these sources the lower limit for the fraction of objects with $N_{\mathrm{H}}<10^{22} \mathrm{~cm}^{-2}$ still remains around $10 \%$, while for only those with known $N_{\mathrm{H}}$ (19) the fraction is $\sim 26 \%$. If we limit the sample in distance to $<22 \mathrm{Mpc}$, in order to limit the number of sources without a column density measurement, we obtain a sample of 27 sources and 14 objects have an estimation of the $N_{\mathrm{H}}$. In this case, the lower limit for the fraction increases to $15 \%$, while for the 14 sources this fraction is $\sim 28 \%$. In Table 3 we show the lower limits for the column density distributions derived in the three samples.

Although the number of sources for which there is no information available is high in all samples, the agreement found is reassuring. It is also interesting to note that an important fraction of unabsorbed objects is found only when low luminosity AGNs (LLAGNs) are considered: this is evident in the Risaliti et al. (1999) total sample when objects at low luminosity are included and it is obvious in the Ho et al. (1997) sample which is mainly made up by such sources.

Recently, Mainieri et al. (2002) have presented the results of the X-ray spectral analysis of the deep survey of the Lockman Hole field with the XMM-Newton observatory. Nearly $30 \%$ of type 2 Seyfert galaxies show $N_{\mathrm{H}}<10^{22} \mathrm{~cm}^{-2}$.

If confirmed by future studies, the fraction of low absorbed Seyfert 2 galaxies obtained (presumably in the range of $10 \%-$ $30 \%$ ) could have important implications for synthesis models of the X-ray background and the Unified Theory of Seyfert galaxies.

In Fig. 4 we plot the column density, measured from the $\mathrm{X}$-ray spectra, against 2-10 keV luminosity corrected for absorption. As expected we find no significant correlation (at $90 \%$ level for a two tailed test) between these two quantities confirming previous findings by Risaliti et al. (1999). Nevertheless it is interesting to note that we have extended the parameter space in Fig. 4 considerably towards lower $N_{\mathrm{H}}$ and X-ray luminosities compared to Risaliti et al. (1999) work: this further supports the idea that more unobscured Seyfert $2 \mathrm{~s}$ are observed going towards low luminosities. Since the LLAGNs constitute the majority of the nearby AGN population, it becomes evident that unobscured type 2 objects may constitute a non-negligible part of the type 2 class. 


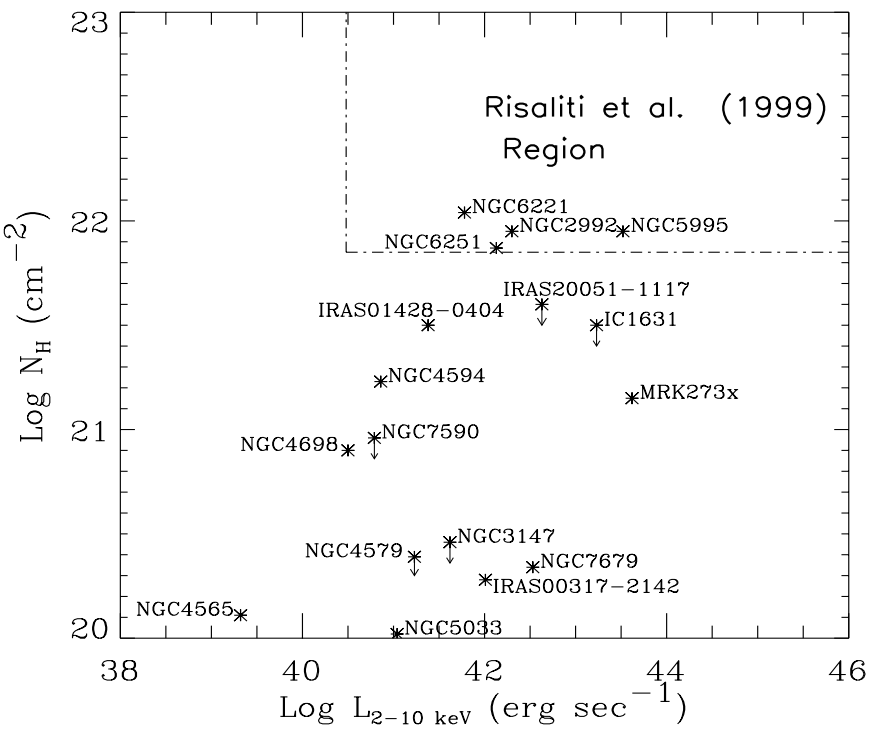

Fig. 4. X-ray column density vs. $L_{2-10} \mathrm{kev}$ for the sources sample. No significant correlation between the two quantities is found. We highlight the region covered by Risaliti et al. (1999) sample sources and show that most of our objects are LLAGNs.

\section{What kind of objects are unabsorbed Seyfert 2s?}

None of the sources in our sample show evidence of the broad $\left(F W H M>1000 \mathrm{~km} \mathrm{~s}^{-1}\right)$ permitted emission lines observed in Seyfert 1s. However, some of our sources show a broad $\mathrm{H}_{\alpha}$ component (NGC 2992, Gilli et al. 2000; NGC 4579, Ho et al. 1997; NGC 4594, Faber et al. 1997; NGC 5995, Lumsden \& Alexander 2001; NGC 7679, Kewley et al. 2000); this is sometime variable (NGC 5033, Ho et al. 1995) and sometime extremely weak (IRAS 00317-2142, Coziol et al. 1993; NGC 4565, Ho et al. 1997).

Polarized spectroscopic measurements are available only for three objects: for both NGC 2992 (Rix et al. 1990) and NGC 5995 (Lumsden \& Alexander 2001; Tran 2001) a polarized broad $\mathrm{H}_{\alpha}$ line has been measured, while for NGC 7590 no polarized broad $\mathrm{H}_{\alpha}$ component has been detected (Heisler et al. 1997).

We have indication that at least 8 sources have broad components, but the broad components are not necessarely associated with an AGN Broad Line Region since they are too narrow for BLR standards. It is likely that the unabsorbed Seyfert 2 galaxies host a hidden BLR but we cannot rule out that in some of these objects the BLR is intrinsecally weak or absent. Therefore two scenarios are plausible:

- A standard BLR exists, however to provide a type 2 classification the BLR must be hidden by a non-standard absorbing medium.

- The BLR is non-standard (very weak or fading away) or it does not exist at all, in this case no absorbing material is required and our sources are truly not absorbed.

A possible solution within the first scenario is to assume a gas-to-dust ratio different from the Galactic one. This is a reasonable assumption since it has already been shown to be the case for a number of Seyfert galaxies (Maiolino et al. 2001). However, contrary to previous studies, in our case we need a dust-to-gas ratio higher than Galactic: in fact galaxies with $N_{\mathrm{H}} \sim 10^{21} \mathrm{~cm}^{-2}$ would have an extinction $A_{V} \sim 0.45$ insufficient to hide the BLR unless the $A_{V} / N_{\mathrm{H}}$ is a factor $10-50$ higher than the Galactic value. This points to dust properties in our galaxies which are anomalous with respect to the great majority of AGNs. This result confirms previous findings of Maiolino et al. (2001) who notice that a few LLAGNs in their sample were characterized by an $A_{V} / N_{\mathrm{H}}$ ratio consistent with or even higher than the Galactic value.

Indeed, in the HST imaging survey of the nearest Seyferts, Malkan et al. (1998) found that the center regions of Seyfert 2 galaxies are intrinsically more dusty than those of Seyfert 1 galaxies, this property manifests itself in the form of lanes and patches that are irregular and reach close to the nucleus. However, this dusty and irregular environment is likely to be located on larger scale than the BLR and is likely to be associated with the inner regions of the host galaxy (Galactic Dust Model, GDM).

Another large scale structure which could be responsible for the absorption measured in X-rays is a starburst region (Weaver 2001; Levenson et al. 2001a, Starburst Model, SBM). Such regions can have large X-ray column densities of up to a few times $10^{22} \mathrm{~cm}^{-2}$. Such values can obscure the central AGN at optical wavelengths making a Seyfert 1 galaxy into a Seyfert 2 without requiring a pc-scale torus. This model also explains a higher presence of dust lanes, bars and star-forming activity in type 2 Seyferts than in type 1 Seyferts (Maiolino et al. 1997). Some objects of our sample are indeed characterized by the presence of a bar (IRAS 00317-2142, IRAS 014280404, NGC 4579, NGC 5995, NGC 6221 and NGC 7679) or emission from an HII region comparable to those of starburst galaxies (IC 1631, NGC 3147 and IRAS 20051-1117) and can therefore be explained within the GDM and SBM.

We can therefore check if the absorption present in our Seyfert 2 sample is compatible with these two models. This is feasible since in our objects the optical extinction at larger scales (i.e. in the NLR) can be assessed from the narrow emission lines using the narrow $\mathrm{H}_{\alpha} / \mathrm{H}_{\beta}$ ratios and applying the Ward et al. (1987) formula:

$A_{V}=6.67 \times\left(\log \left(\mathrm{H}_{\alpha} / \mathrm{H}_{\beta}\right)-\log (2.85)\right) \quad(\mathrm{mag})$.

These values can then be translated into gas column densities using Eq. (2) from Gorenstein (1975) and compared to the $N_{\mathrm{H}}$ values measured from X-ray data to obtain the $A_{V} / N_{\mathrm{H}}$ ratio relative to the NLR. This $A_{V} / N_{\mathrm{H}}$ distribution is plotted in Fig. 5 where it is evident that the obscuration seen in X-rays is consistent with that measured in the NLR, expecially after considering the large errors affecting both the optical and X-ray measurements.

This conversely implies that in our sources the obscuring torus is either not visible or not present. This result conforms with both the GD and SB models in the sense that the absorbing material in X-rays is located at larger scale and that the $A_{V} / N_{\mathrm{H}}$ ratio measured in our sources allow for a dust-to-gas ratio equal to or even higher than Galactic. In this second hypothesis the 


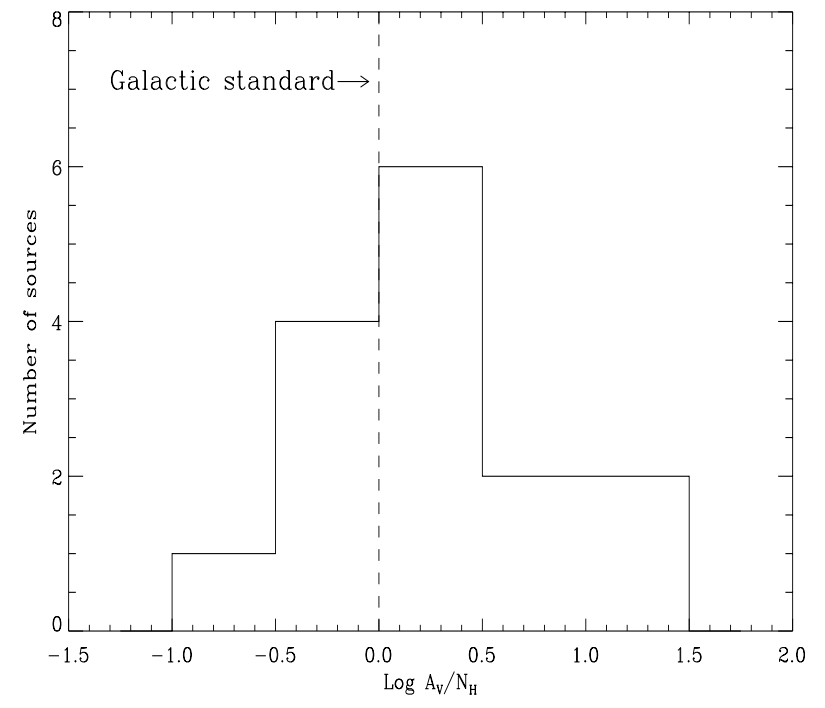

Fig. 5. $\log A_{V} / N_{\mathrm{H}}$ distribution for the sample. The dashed line is relative to the standard Galactic value.

optical extinction could be sufficient to hide the BLR and provide the type 2 classification of our sources.

In the second scenario no absorption is required to hide the broad line region which is therefore absent or very weak. This scenario is more likely applicable to low luminosity AGNs where the brightness of the active nucleus may be insufficient to photoionize the BLR.

\section{Discussion}

Under the Unified Theory hypothesis, when we are looking at a Seyfert 2 we should expect to measure a high X-ray column density produced by a thick molecular torus which hides the BLR. According to the results detailed above, the column density measured in our Seyfert sample is consistent with the extinction obtained from the reddening of the lines produced in the NLR. This means that the presence of an obscuring torus is not required and, therefore, that the objects considered in our sample are somehow anomalous Seyfert 2 galaxies. A column density of $N_{\mathrm{H}} \sim 10^{22} \mathrm{~cm}^{-2}$ or slightly under this value can be associated with the presence of a dust lane, a bar or an HII region. This could be the case for the objects in the present sample which in Fig. 4 populate the high X-ray luminosity - high column density region (IRAS 01428-0404, IC 1631, NGC 2992, NGC 5995, NGC 6221, NGC 6251 and IRAS 20051-1117); these have $N_{\mathrm{H}}$ values sufficient to cover the BLR. These objects could be seen perpendicularly to the torus and some other type of absorbing material obscures the BLR. To summarise, if the BLR of these galaxies is obscured, then a larger-scale dusty environment is likely to be responsible for their obscuration.

However, in Fig. 4 at lower X-ray luminosities and lower column densities we find those objects with $N_{\mathrm{H}}$ very low and sometimes compatible with the Galactic absorption; i.e. a value not sufficient to obscure the BLR (IRAS 00317-2142, NGC 3147, NGC 4579, NGC 4594, NGC 4565, NGC 4698, NGC 5033, MRK 273x, NGC 7590 and NGC 7679).
A dust-to-gas ratio different from the Galactic one could be a possible solution to the problem, i.e. a strong dust contribution obscures the BLR and it is not detectable in X-rays. It is worth noting that, except for MRK 273x and NGC 7679, these low $N_{\mathrm{H}}$ Seyferts are all LLAGNs. The nature of LLAGNs is a hotly debated issue, since it is not clear what powers such objects and it is not obvious that the unified model is valid at these low luminosities (Ho et al. 2001).

A few of our galaxies have very massive black holes ( $>10^{9} M_{\odot}$ for NGC 4594 and $6-13 \times 10^{7} M_{\odot}$ for NGC 4579) and they accrete at extremely sub-Eddington rates $\left(L / L_{\text {Edd }}<\right.$ $10^{-5}$, Ho 1999). It is possible that in these extreme conditions there is not sufficient ionization power to illuminate the BLR or there is insufficient gas to feed the clouds in this region or the structure of the accretion flow is uncapable of activating it (Barth 2002). Such objects would be preferentially detected in early type galaxies with massive black holes because a subEddington accreting AGN would be too faint to be visible in a spiral with a low black hole mass. About $50 \%$ of our LLAGNs are in early type galaxies. There is observational evidence to support the above suggestion: several recent works have investigated the size-mass-luminosity relations in AGNs (Wandel et al. 1999; Kaspi 2000; Collin \& Huré 2001) supporting the idea that there is a correlation between the luminosity of the AGN and its BLR size in the sense that the BLR size decreases as the AGN luminosity decreases.

There are also theoretical studies which lead us to believe that in intrinsically weak AGNs the BLR is extremely faint or absent. Nicastro (2000) suggests a model in which the FWHMs of the broad emission lines are related to the accretion rate ${ }^{2}$, in particular at very low accretion rates the clouds of the BLR would cease to exist. Therefore for sufficiently weak AGNs no BLR is expected. This is the "pure" Seyfert 2 model (Tran 2001).

Furthermore, we consider the hypothesis of "Fossil" objects in which the central engine has turned off (Maiolino 2001). Shortly after this occurs, the BLR fades away and the nucleus appears as a type 2 object. If the nucleus remains quiescent for a sufficient time then even the echo of the NLR will fade away. Under this hypothesis unabsorbed Seyfert 2 s are simply a transition state of an AGN. Since this phase should rapidly decay, the fraction of fossil AGNs may be very small.

The obscuring material around an active nucleus has been proven by some recent evidence to be complex and variable and the possibility that in some objects our line-of-sight might be intercepting a "hole" in the absorber at a certain time must also be taken into account (Risaliti 2002).

To summarize, in the 17 unabsorbed Seyfert 2 galaxies the low absorption is not likely to be related to the torus but to material distributed over larger scale, very likely the NLR unless the BLR is particularly dusty. Some of these objects could be

\footnotetext{
2 In this model the broad emission lines originate in a vertical disk wind, at a critical distance in the accretion disk and the widths of such broad emission lines are the Keplerian velocities of the accretion disk at the radius at which the wind originates. The disk wind forms for external accretion rates higher than a minimum value below which a standard disk is stable.
} 
seen perpendicularly to the torus with some types of absorbing material obscuring the BLR; the material may be slightly dusty and associated with lanes, patches or starburst regions. In a few cases, the lack of evidence for the existance of a BLR suggests that this is weak, absent or has just faded away. In this case the nucleus is of low luminosity and this may explain the weakness or lack of a BLR.

\section{Conclusions}

We present a sample of type 2 Seyfert galaxies which behave differently than classical type 2 objects. We have shown that there are strong indications in favour of the "true" Compton thin nature of such sources.

We estimate the fraction of Seyfert $2 \mathrm{~s}$ with low absorption to be in the range of $10 \%-30 \%$ and this fraction is likely to increase at low luminosities. The study of a complete sample of LLAGNs will provide a more reliable estimate of their incidence in the Seyfert galaxies population.

The unabsorbed Seyfert 2 galaxies cannot be described by the Unified Model in its simple formulation. The standard BLR of Seyfert galaxies is either obscured by something other than the torus or it is weak, absent or has just faded away.

As a concluding remark, it is worth noting that the results presented in this paper add further complications to the classification scheme of AGNs in general and of Seyfert galaxies in particular.

Acknowledgements. We thank Massimo Cappi and Luigi Foschini for useful comments and John Stephen for the help in the final revision. We thank the referee for the helpful comments that improved the manuscript. This work has made use of XMM-Newton data. This research has made use of the NASA/IPAC extragalactic database (NED) which is operated by the Jet Propulsion Laboratory, California Institute of Technology, the High Energy Astrophysics Science Archive Research Center (HEASARC) provided by NASA's Goddard Space Flight Center, the BeppoSAX Science Data Center, the ASCA archive and the TARTARUS database.

\section{References}

Allen, S. W., Di Matteo, T., \& Fabian, A. C. 2000, MNRAS, 311, 493 Antonucci, R. R. J., \& Miller, J. S. 1985, ApJ, 297, 621

Antonucci, R. R. J. 1993, ARA\&A, 31, 473

Awaki, H. 1992, in UAP 103, Proc. of Frontieres of X-ray Astronomy, ed. Y. Tanaka, \& K. Koyama

Barth, A. J. 2002, to appear in Issues in Unification of AGNs, ed. R. Maiolino, A. Marconi, \& N. Nagar, ASP Conf. Ser., 258

Bassani, L., Dadina, M., Maiolino, R., et al. 1999, ApJS, 121, 473

Cappi, M., Di Cocco, G., Panessa, F., et al. 2002, to appear in the Proc. Symp. New Visions of the X-ray Universe in the XMM-Newton and Chandra Era, 26-30 November 2001, ESTEC, The Netherlands, ASP Conf. Ser. 9 [astro-ph/0202245]

Collin, S., \& Huré, J.-M. 2001, A\&A, 372, 50

Coziol, R., Demers, S. P. M., Torres-Peimbert, S., et al. 1993, AJ, 105, 35

David, L. P., Jones, C., \& Forman, W. 1992, ApJ, 388, 82

Della Ceca, R., Pellegrini, S., Bassani, L., et al. 2001, A\&A, 375, 781

Eracleous, M., Shields, J. C., Chartas, G., \& Moran, E. C. 2001, AAS, 19914103E

Faber, S. M., Tremaine, S., Ajhar, E. A., et al. 1997, AJ, 114, 1771
Georgantopoulos, I. 2000, MNRAS, 315, 77

Gilli, R., Maiolino, R., Marconi, A., et al. 2000, A\&A, 355, 485

Gorenstein, P. 1975, ApJ, 198, 95

Guainazzi, M. 2002, MNRAS, 329, L13

Heckman, T. M. 1980, A\&A, 87, 152

Heisler, C. A., Lumsden, S. L., \& Bailey, J. A. 1997, Nature, 385, 700

Ho, L. C., Filippenko, A. V., \& Sargent, W. L. 1995, ApJS, 98, 477

Ho, L. C., Filippenko, A. V., \& Sargent, W. L. W. 1997, ApJS, 112, 315

Ho, L. C. 1999, ApJ, 516, 672

Ho, L. C., Feigelson, E. D., Townsley, L. K., et al. 2001, ApJ, 549, 51

Kaspi, S., Smith, P. S., Netzer, H., et al. 2000, ApJ, 533, 631

Kewley, L. J., Heisler, C. A., Dopita, M. A., et al. 2000, ApJ, 530, 704

Levenson, N. A., Weaver, K. A., \& Heckman, T. M. 2001a, ApJ, 550, 230

Levenson, N. A., Cid Fernandes, R., Weaver, K. A., et al. 2001b, ApJ, in press

Lumsden, S. L., \& Alexander, D. M. 2001, MNRAS, 328, 32

Mainieri, V., Bergeron, J., Hasinger, G., et al. 2002, A\&A, 393, 425

Maiolino, R., \& Rieke, G. H. 1995, ApJ, 454, 95

Maiolino, R., Ruiz, M., Rieke, G. H., \& Papadopoulos, P. 1997, ApJ, 485,552

Maiolino, R. 2001, Invited talk at the conference X-ray Astronomy: Stellar Endpoints, AGN and the Diffuse X-ray Background, held in Bologna, Italy, 6-10 Sep. 1999, ed. N. E. White, G. Malaguti, \& G. G. C. Palumbo, AIP Conf. Proc., 599, 199

Maiolino, R., Marconi, A., \& Oliva, E. 2001, A\&A, 365, 37

Malkan, M. A., Gorjian, V., \& Tam, R. 1998, ApJS, 117, 25

Moran, E. C., Halpern, J. P., \& Helfand, D. J. 1996, ApJS, 106, 341

Moran, E. C., Barth, A. J., Kay, L. E., \& Filippenko, A. V. 2000, ApJ, 540,73

Mulchaey, J. S., Koratkar, A., Ward, M. J., et al. 1994, ApJ, 436, 586

Nicastro, F. 2000, ApJ, 530L, 65N

Pappa, A., Georgantopoulos, I., Stewart, G. C., \& Zezas, A. L. 2001, MNRAS, 326, 995

Pellegrini, S., Fabbiano, G., Fiore, F., Trinchieri, G., \& Antonelli, A. 2002, A\&A, 383, 1

Pietsch, W., Bischoff, K., Boller, Th., et al. 1998, A\&A, 333, 48

Ptak, A., Yaqoob, T., Serlemitsos, P. J., Kunieda, H., \& Terashima, Y. 1996, ApJ, 459, 542

Risaliti, G., Maiolino, R., \& Salvati, M. 1999, ApJ, 522, 157

Risaliti, G. 2002, A\&A, 386, 379

Rix, H., Rieke, G., Rieke, M., \& Carleton, N. P. 1990, ApJ, 363, 480

Sambruna, R. M., Eracleous, M., \& Mushotzky, R. F. 1999, ApJ, 526, 60

Sekiguchi, K., \& Wolstencroft, R. D. 1993, MNRAS, 263, 349

Shuder, J. M., \& Osterbrock, D. E. 1981, ApJ, 250, 55

Smith, D. A., \& Done, C. 1996, MNRAS, 280, 355

Terashima, Y., Ho, L. C., Ptak, A. F., et al. 2000, ApJ, 533, 729

Tran, H. D. 2001, ApJ, 554, 19

Turner, T. J., George, I. M., Nandra, K., \& Mushotzky, R. F. 1997, ApJS, 113, 23

Vaceli, M. S., Viegas, S. M., Gruenwald, R., \& de Souza, R. E. 1997, AJ, 114, 1345

Veilleux, S., \& Osterbrock, D. E. 1987, ApJS, 63, 295

Xia, X.-Y., Mao, S., Wu, H., et al. 1999, ApJ, 524, 746

Xia, X. Y., Xue, S. J., Mao, S., et al. 2002, ApJ, 564, 196

Wandel, A., Peterson, B. M., \& Malkan, M. A. 1999, ApJ, 526, 579

Ward, M. J., Geballe, T., Smith, M., Wade, R., \& Williams, P. 1987, ApJ, 316, 138

Weaver, K. A., Two Years of Science with Chandra, Abstracts from the Symp. held in Washington, DC, 5-7 Sep., 2001, Invited Talk, 2001, tysc.conf.1004W 